\title{
Investigation of dispersion and nonlinear characteristics of liquid core optical fiber filled with olive oil
}

\author{
Anwaar A. Al-Dergazly ${ }^{1^{*}}$ \\ ${ }^{1}$ Laser and Optoelectronics Engineering Department, College of Engineering, Nahrain University, Baghdad, Iraq
}

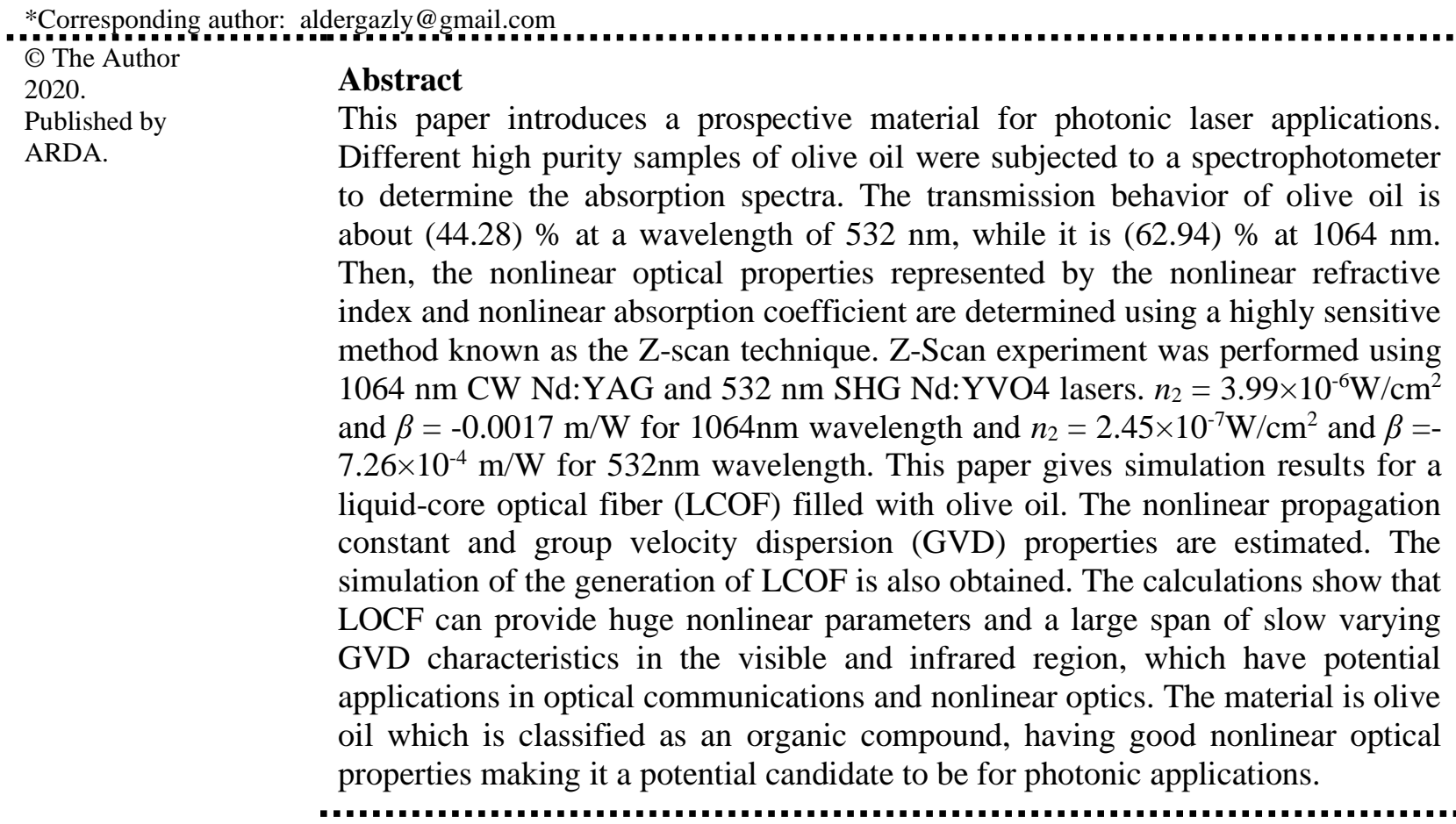

Keywords: Chromatic dispersion, GVD, Nonlinear coefficient, Organic materials, Optical fiber

\section{Introduction}

Organic materials have been used in nonresonant photonic applications due to their high nonlinear coefficients $\gamma$, high damage threshold and good transparency. Olive oil behaves as a nonlinear material where it has a "blue shift" in some excited wavelengths [1]. Therefore, the nonlinear optical properties of olive oil which are of prominent importance for photonics applications are investigated in this work.Lyotropic liquid crystals (LLC) are mixtures of amphiphilic molecules and a solvent self- assembled in aggregates at certain condition of temperature and relative concentrations [2]. Besides, LOCF has advantage of easy fabrication and robustness compared with silica nano-wire. The olive oil represents the one class of lyotropic liquid crystal in nematic phase [3]. When applied optical field to the Liquid crystals the behavior of the crystals appear as nonlinear optic crystal materials. The polarized light beam from laser source can induce an alignment or ordering in isotropic phase. These result in a change in refractive index. In isotropic phase the change in refractive index is due to the density change following a rise in temperature. In the nematic phase the change in the refractive index depend directly on the temperature. The reorientation of molecules in liquid crystals depends on the phase of liquid crystals. For the isotropic phase the liquid crystal molecules are randomly oriented owing to

This work is licensed under a Creative Commons Attribution License (https://creativecommons.org/licenses/by/4.0/ ) that allows others to share and adapt the material for any purpose (even commercially), in any medium with an acknowledgement of the work's authorship and initial publication in this journal. 
thermal motion. So, an intense laser field will force the anisotropic molecules to align themselves in the direction of optical field through the dipolar interaction.

\section{Method}

\subsection{Fiber losses}

One of the important fibers parameters is the measure of power loss during transmission of optical signals inside the fiber. Several factors contribute to the losses of conventional fibers, with material absorption and Rayleigh scattering as a dominating contribution. In photonic crystal fiber (PCFs), there is another source of losses which is called confinement losses. The origin behind this type of losses is that the core material is made from (silica) and therefore it is difficult to confine the light in the core without increasing the number of hole rings in the cladding layer [4]. Consider the simplified case of propagating a monochromatic optical wave in a fiber extended along the $z$ axis. When fiber dispersion, optical nonlinearity, and boundary conditions are neglected, the general form of the electric field for the optical wave in the fiber can be written as [5]:

$$
\begin{aligned}
& E(t, z)=A_{o}\left(e^{-\propto \mathrm{z} / 2}\right) \cos (\omega t-\beta z) \\
& =\operatorname{Re}\left[A_{o} e^{(-\propto \mathrm{z} / 2)} \cdot e^{-j(\omega t-\beta z)}\right]
\end{aligned}
$$

where, $\operatorname{Re}\left[\right.$ ] stands for the real part of the argument, $A_{o}=$ Field amplitude at $z=0, \alpha=$ Fiber optical loss coefficient, $t=$ Time, $\omega=$ angular frequency of the optical wave, $\beta$ is Propagation constant (wave number). The propagation constant $\beta=2 \pi n / \lambda$ where $\lambda$ is the optical wavelength. Then Eq. (1) can be written as:

$$
E(t, z)=\operatorname{Re}\left[A_{0} \cdot e^{j(\omega t-2 \pi \bar{n} z / \lambda)}\right]
$$

where, $n$ is the complex refractive index

$\bar{n}=n-j \frac{\alpha \lambda}{4 \pi}$

Thus, $\alpha$ is related to the imaginary part of the complex refractive index

$$
\alpha=-\frac{4 \pi}{\lambda} \operatorname{Im}[\bar{n}]
$$

The optical power is proportional to the square of the electric field amplitude E.

\subsection{Fiber chromatic dispersion}

Chromatic dispersion is the phenomenon wherein different spectral components of the transmitted laser signal travel at different velocities in the fiber, arriving at different times at the receiver. Depending on system length and bit rate per wavelength, the amount of chromatic dispersion in a system must be carefully managed with dispersion compensators to achieve the right amount of residual dispersion [6].

Chromatic dispersion plays a critical role in propagation of short optical pulses in single-mode fibers because different spectral components associated with the pulse travel at different speeds given by $c / n(\omega)$ where $\mathrm{c}$ is the speed of light in vacuum. Thus, the origin behind chromatic dispersion is the frequency dependence of the refractive index $n(\omega)$. The mode-propagation constant becomes frequency dependent according to [7,8]. 


$$
\beta(\omega) \equiv \frac{2 \pi}{\lambda} n(\omega)=\frac{\omega}{c} n(\omega)
$$

Expanding the mode-propagation constant in a Taylor series about the frequency $\omega_{o}$ at which the pulse spectrum is centered [9]:

$$
\beta(\omega)=\beta_{o}+\beta_{1}\left(\omega-\omega_{o}\right)+\frac{1}{2} \beta_{2}\left(\omega-\omega_{o}\right)^{2}+\cdots
$$

where,

$$
\beta_{m}=\left(\frac{d^{m} \beta}{d \omega^{m}}\right)_{\omega=\omega_{o}} \quad(m=0,1,2, \ldots \ldots)
$$

The parameters $\beta_{1}$ and $\beta_{2}$ are related to the refractive index $\mathrm{n}$ and its derivatives through the relations

$$
\begin{aligned}
& \beta_{1}=\frac{1}{v_{g}}=\frac{n_{g}}{c}=\frac{1}{c}\left(n+\omega \frac{d n}{d \omega}\right) \\
& \beta_{2}=\frac{1}{c}\left(2 \frac{d n}{d \omega}+\omega \frac{d^{2} n}{d \omega^{2}}\right)
\end{aligned}
$$

where, $n_{g}$ is the group index and $v_{g}$ is the group velocity. Note that the envelope of the optical pulse moves at the group velocity while the parameter $\beta_{2}$ represents dispersion of the group velocity and is responsible for pulse broadening.

The group-velocity dispersion (GVD) parameter $\beta_{2}$ is related to the dispersion parameter $\mathrm{D}$ that is commonly used in the optical communication literature. This quantity is usually specified with units of $\mathrm{ps} /(\mathrm{nm} \mathrm{km})$ (picoseconds per nanometer wavelength change and kilometer propagation distance) $[7,8]$.

$$
D=\frac{d \beta_{1}}{d \lambda}=-\frac{2 \pi c \beta_{2}}{\lambda^{2}} \cong-\frac{\lambda}{c} \frac{d \mathrm{n}^{2}}{d \lambda^{2}}
$$

The forgoing discussion highlights the concepts behind material dispersion which is due to the frequency dependence of the refractive index of the material of the fiber core. However, because of dielectric wave guiding, the effective mode index is slightly lower than the material index $n_{\omega}$ of the core, the reduction itself being frequency dependent. This results in a waveguide contribution that must be added to the material contribution to obtain the total dispersion. In standard single-mode fibers, the total second-order dispersion D vanishes at $\lambda=1300 \mathrm{~nm}$ [6]. Pulse propagation near this wavelength requires inclusion of the third-order dispersion (TOD) parameter $\beta_{3}$ or equivalently inclusion of the slope of GVD,

$S=\frac{d D}{d \lambda}=\frac{d^{2} \beta_{1}}{d \lambda^{2}}=\left[\frac{4 \pi c \beta_{2}}{\lambda^{3}}-\frac{2 \pi c}{\lambda^{2}} \frac{d \beta_{2}}{d \lambda}\right]$

When short optical pulses propagate inside a fiber, both dispersive and nonlinear effects influence their shape and spectrum. The pulse propagation is described by the nonlinear Schrodinger (NLS) Equation $[9,10]$

$$
\frac{\partial A}{\partial z}+\beta_{1} \frac{\partial A}{\partial z}+\frac{j \beta_{2}}{2} \frac{\partial^{2} A}{\partial t^{2}}-\frac{\beta_{3}}{6} \frac{\partial^{3} A}{\partial t^{3}}+\frac{\alpha}{2} A=j \gamma|A|^{2} A
$$

where $A(t, z)$ is the slowly varying envelope of the electric field and $\gamma$ is the nonlinear parameter 


$$
\gamma=\frac{n_{2} \omega_{o}}{c A_{e f f}}
$$

$\omega_{o}$ is the center frequency of the pulse spectrum, and $A_{\text {eff }}$ is the effective core area.

\section{Results and discussion}

\subsection{Olive oil spectrum and refractive index measurements}

The refractive index of silica and olive oil vs. wavelength were calculated using Sellmeier Equation [11] as shown in Fig. 1.

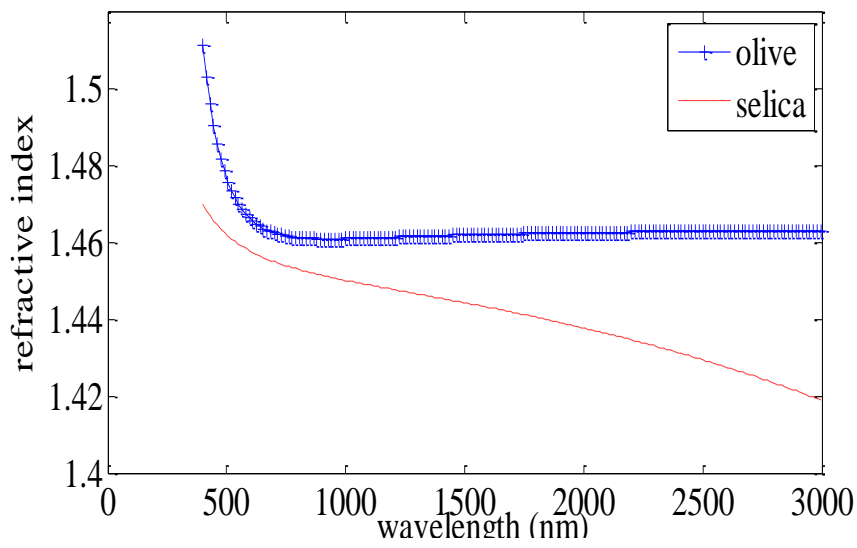

Figure 1. Refractive index versus wavelengths (nm) for olive oil and silica

Fig. 1 shows that the refractive index of the olive oil is larger than the refractive index of silica so it is achieved with total internal reflection $n_{\text {core }}>n_{\text {cladding }}$

The experimental work is based on testing the olive oil transmission spectrum using UV-VIS spectrophotometer. Fig. 2 shows the transmission spectrum of olive oil. The optical transmission of olive oil shows a variable behavior of the transmission as a function of the incident wavelength. The transmission behavior of olive oil is about (44.28) \% at wavelength of $532 \mathrm{~nm}$, while it is (62.94) \% at $1064 \mathrm{~nm}$ as shown in Fig. 2. The transmission spectrum shows the maximum peak at $(850) \mathrm{nm}$.

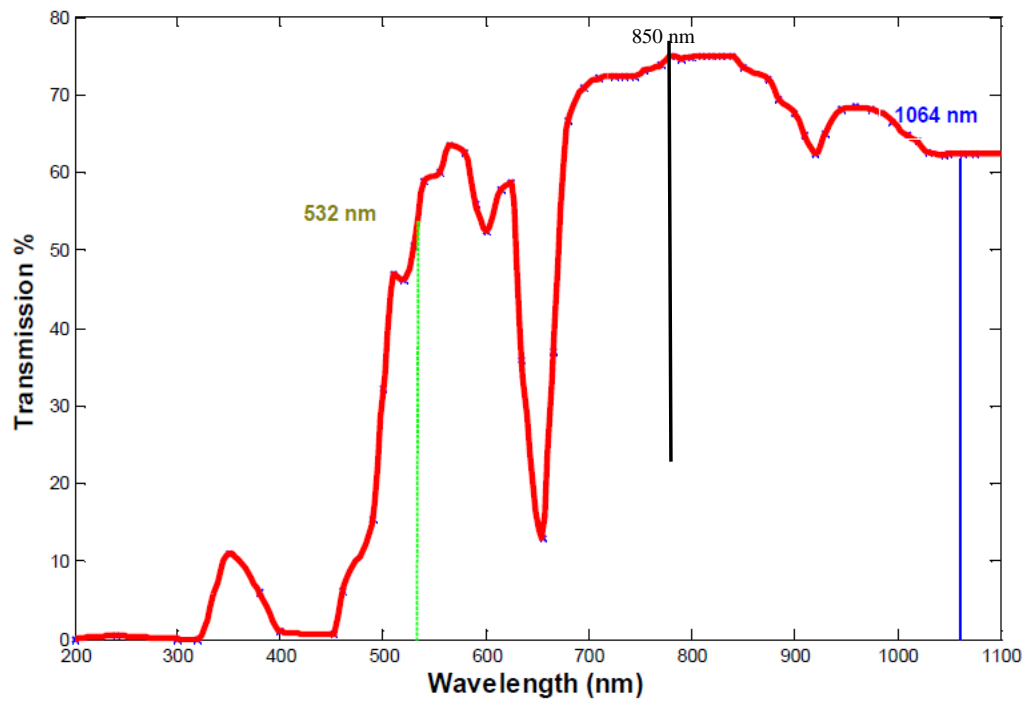

Figure 2. UV-VIS transmission of olive oil 


\subsection{Dispersion measurements}

The parameters $\beta_{2}, \beta_{3}$ and $\beta_{4}$, were determined using Eq. (7) for olive oil related to the refractive index $\mathrm{n}$ as show in Fig. 3,4 and 5. This results in a waveguide contribution that must be added to the material contribution to obtain the total dispersion.

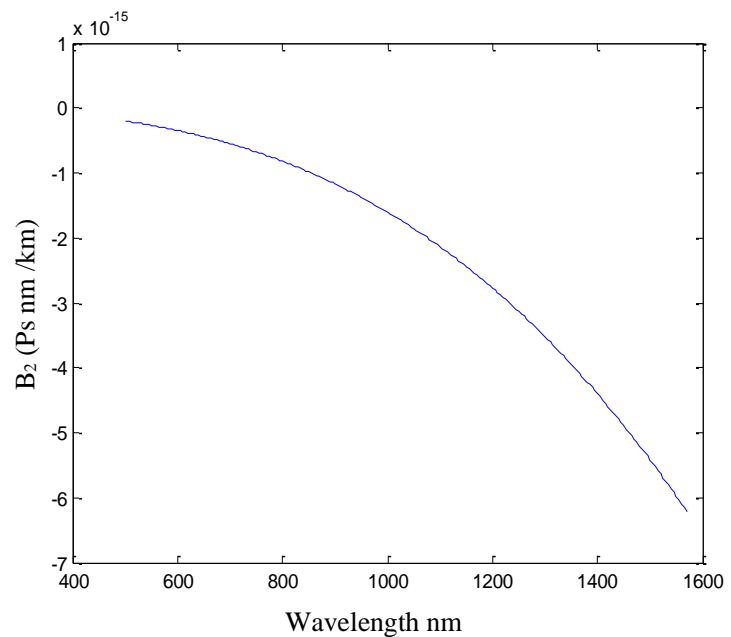

Figure 3. Group-velocity dispersion $B_{2}$

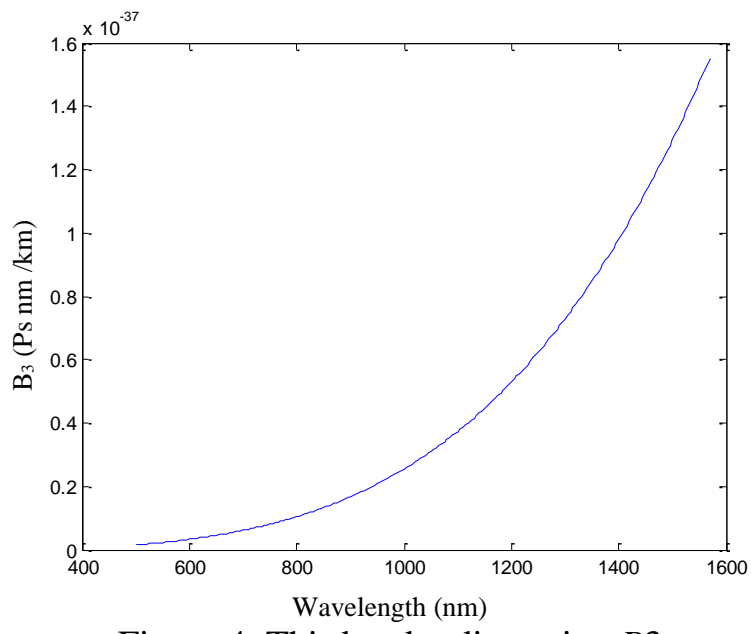

Figure 4. Third-order dispersion $B 3$

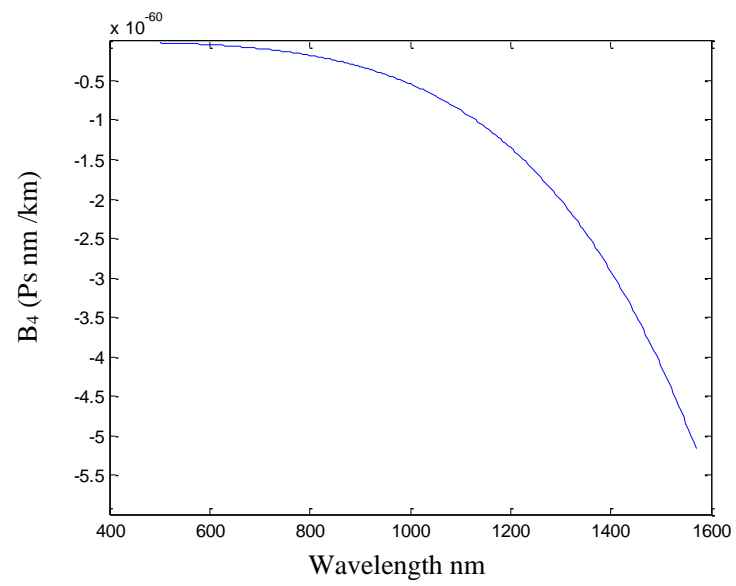

Figure 5. Fourth order dispersion $B_{4}$

Dispersion parameter D that is commonly used in the optical communication was calculated from Eq. (8) and display in Fig. 6. The slope of GVD (S) was determine from Eq. (9) as shown in Fig. 7

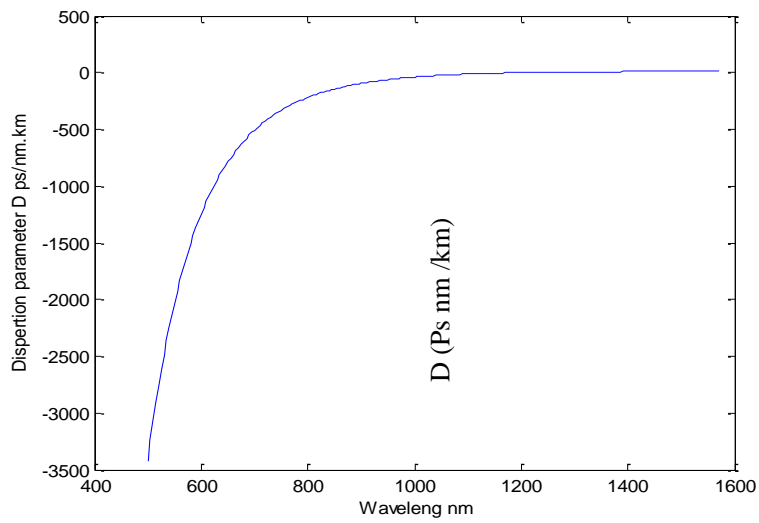

Figure 6. Dispersion parameter $D$

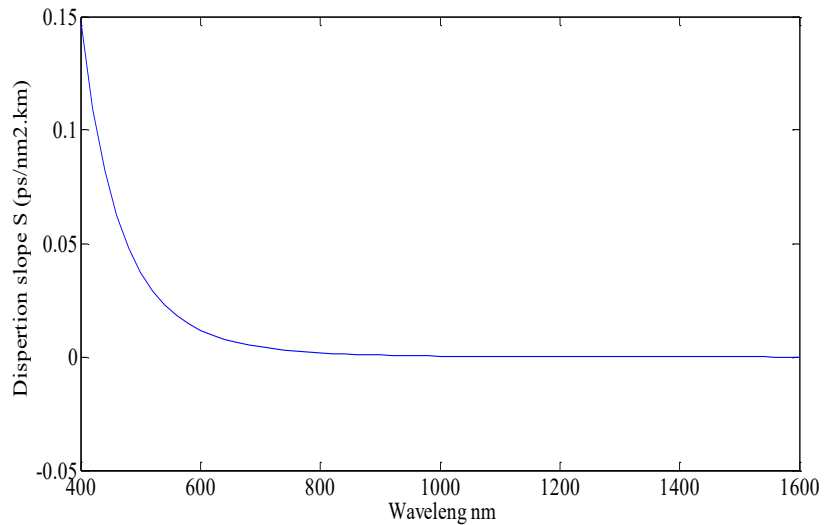

Figure 7. Chromatic dispersion slope 


\subsection{Nonlinear parameter of olive oil}

Using Z-scan techniques [12] to determine the nonlinear refractive index and nonlinear absorption coefficient of the olive oil. The nonlinear refractive index of olive oil $n_{2}$ versus input powers and wavelengths is determined which are equal $n_{2}=3.99 \times 10^{-6} \mathrm{~W} / \mathrm{cm}^{2}$ for $1064 \mathrm{~nm}$ wavelength and $n_{2}=2.45 \times 10^{-7} \mathrm{~W} / \mathrm{cm}^{2}$ for $532 \mathrm{~nm}$ wavelength.

Olive oil has a saturation effect for both wavelengths $532 \mathrm{~nm}$ and $1064 \mathrm{~nm}$ in $\mathrm{CW}$ regime. The nonlinear absorption coefficients are $\beta=-0.0017 \mathrm{~m} / \mathrm{W}$ for $1064 \mathrm{~nm}$ and $\beta=-7.26 \times 10^{-4} \mathrm{~m} / \mathrm{W}$ for $532 \mathrm{~nm}$ wavelength.

The nonlinear parameter of olive oil will be determined at $532 \mathrm{~nm}$ and $1064 \mathrm{~nm}$ wavelength for different core diameter using Eq. (11) as shown in Fig. 8 and Fig. 9.

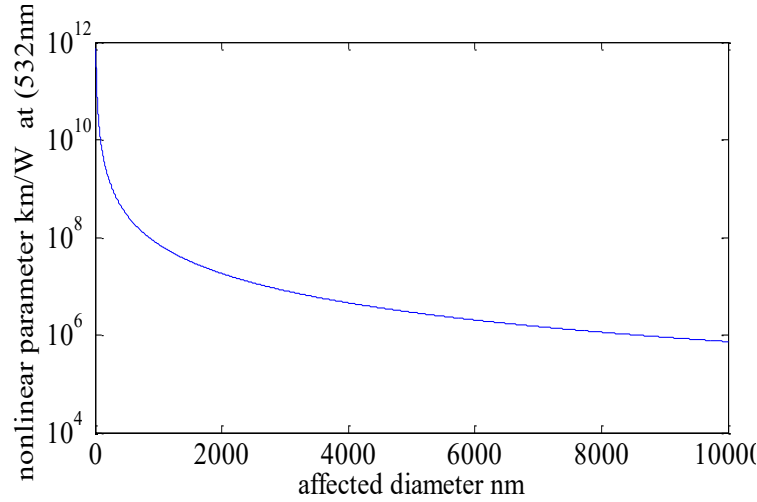

Figure 8. Nonlinear parameter versus core diameter for $532 \mathrm{~nm}$ wavelength

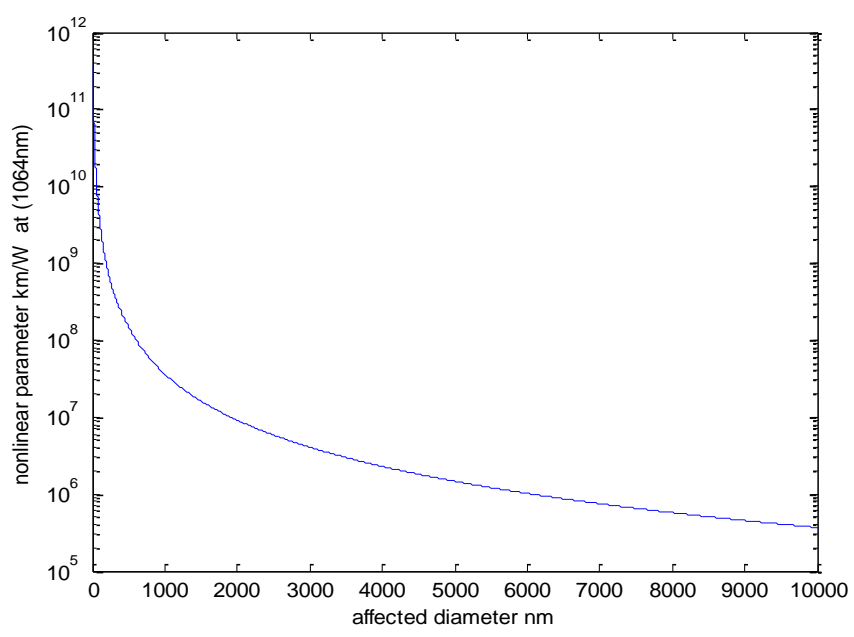

Figure 9. Nonlinear parameter versus core diameter for $1064 \mathrm{~nm}$ wavelength

\section{Conclusion}

- In this paper we have obtained the dispersion parameter of olive oil $\beta_{2}, \beta_{3}, \beta_{4}$, these parameters can be used for many applications like Four Wave Mixing.

- $\quad D$ and $S$ for olive oil will be comparing with the other materials olive oil show lower dispersion that approaches to zero.

The olive oil have a high nonlinear refractive index and nonlinear absorption values compared with other materials (i.e. Toluidine Blue $\mathrm{O}$ dye in Acetonitrile have nonlinear refractive index $\mathrm{n}_{2}=1.4 \times 10^{-7} \mathrm{~W} / \mathrm{cm}^{2}$ and nonlinear absorption coefficient $0.34 \times 10^{-3} \mathrm{~cm} / \mathrm{W}$ ), which can be a promising candidate for photonic device application.

\section{References}

[1] A. A. Al-Dergazly, A. Fadell, "Investi-gation of olive oil as a new photonic liquid crystal for optical communication systems", Proceedings of the 2nd wseas international conference on nanotechnology, Advanced research in physics and engineering, ISSN: 1790-5117, (WSEAS) stevens point, Wisconsin, ISBN: 978-960-474-163-2, USA, 2010.

[2] S.L. G'omez, F.L.S. Cuppo, and A.M. Figureueiredo Neto," Nonlinear optical properties of liquid crystals probed by Z-scan technique", Brazilian Journal of Physics, vol. 33, no. 4, 2003.

[3] G. Burducea, " Lyotropic Liquid Crystals", Romanian Reports in Physics, vol. 56, no. I, pp.66-86, 2004. 
[4] S. M. A. Razzak and Y. Namihira, "Tailoring dispersion and confinement losses of photonic crystal fibers using hybrid cladding", Journal of Lightwave Technology, vol. 26, no. 13, pp. 1909-1914, July 2008.

[5] G. P. Agrawal, "Fiber-optic communication systems", 3ed., John Wiley and Sons, New York, 2002.

[6] A. Walter, G. Schaefer, "Chromatic Dispersion Variations in Ultra-Long-Haul Transmission Systems Arising from Seasonal Soil Temperature Variations", Optical Society of America, USDA/NRCS, National Water and Climate Center, 101 SW Main, Suite 1600, Portland OR 97204 (503) 414-3068 voice, 2001.

[7] A. Akhtar, L. Pavel, and S. Kumar, "Modeling and analysis of the contribution of channel walk-off to nondegenerate and degenerate four-wave mixing noise in RZ-OOK optical transmission systems", IEEE Photonic Technology Letters, vol. 24, no. 11, pp. 4269-4285, November 2006.

[8] H. Li, A. Mafi, A. Schulzgen, L. Li, V. L. Temyanko, N. Peyghambarian, and J. V. Moloney, "Analysis and design of photonic crystal fibers based on an improved effective-index method," Journal of Lightwave Technology, vol. 25, no. 5, pp. 1224-1230, May 2007.

[9] G. P. Agrawal,"Nonlinear fiber optics," Academic Press , $4^{\text {th }}$ edition, 2007.

[10] M. Koshiba, "Full-vector analysis of photonic crystal fibers using the finite element method", IEICE Trans. Electron, vol. E85-C, pp. 881-888, 2002.

[11] R.W. Boyd, G. L. Fischer, Encyclopedia of materials: Scince and Technology, 6237-6244, 2001.

[12] E. Shahriari and W. Mahmood Mat Yunus, "Single Beam Z-Scan Measurements of Nonlinear Refraction and Nonlinear Absorption Coefficients in Silver Nano-Fluid", American J. of Engineering and Applied Sciences, vol. 3, no.1, pp. 98-101, 2010, ISSN 1941-7020, 2010. 